\title{
Determinación preliminar de productos secundarios de cloración (SPD), en aguas crudas del rio Magdalena tratadas con coagulantes naturales $\mathbf{y}$ sintéticos.
}

\section{Preliminary determination of by-products of chlorination (SPD), in raw waters of the river Magdalena treated with coagulant natural and synthetic.}

\author{
Carmen Helena Sampayo Benavides ${ }^{1}$, Jhon Jairo Feria Diaz ${ }^{2}$ \\ ${ }^{1}$ Universidad de Sucre-SUE CARIBE. csampayob@gmail.com \\ ${ }^{2}$ Universidad de Sucre. Grupo GIMAGUAS.jhon.feria@unisucre.edu.co
}

\begin{abstract}
Resumen
El objetivo de este trabajo fue comprobar preliminarmente la presencia de sustancias químicas nocivas como subproductos del uso de coagulantes naturales y sintéticos, en el proceso de coagulación-floculación de agua cruda del río Magdalena, Colombia. Para el efecto, se cuantificó con cromatografía de gases, de acuerdo con metodologías estándar y en un laboratorio validado $y$ acreditado por el IDEAM los Trihalometanos en el agua tratada con coagulante natural (semillas de Moringa oleífera) y sintético (Sulfato de Aluminio tipo B) e hipoclorito de sodio al 5,25\% con dosis óptima de $60 \mathrm{mg} \mathrm{L}^{-1}$. Los resultados de los ensayos mostraron que el agua tratada con coagulante natural superó el máximo permitido de Trihalometanos, de acuerdo con la normatividad sanitaria y ambiental vigente en Colombia. Se concluyó que el coagulante natural, al igual que el sintético, tiene gran capacidad de coagulación del agua cruda del río Magdalena. Sin embargo, no es recomendable su uso simultáneo con cloro, porque da lugar a la producción de cloroformo, que es la principal fuente de Trihalometanos.
\end{abstract}

Palabras clave: Agua potable, Moringa oleífera, Sulfato de Aluminio, Trihalometano.

\begin{abstract}
The objective of this work was the preliminary verification of the presence of chemical substances as the byproducts of the use of natural and synthetic coagulants, in the process of coagulation and flocculation of raw water of the Magdalena river, Colombia. For this purpose, it was quantified with gas chromatography, according to standard methodologies and in a validated laboratory accredited by the IDEAM the Trihalomethanes in the water treated with natural coagulant (Moringa oleifera seeds) and synthetic (Aluminum Sulfate type B) and 5.25\% sodium hypochlorite with an optimum dose of $60 \mathrm{mg} \mathrm{L}-1$. The results of the tests showed that the water treated with natural coagulant exceeded the maximum allowed of Trihalomethanes, in accordance with the sanitary and environmental regulations in force in Colombia. It was concluded that the natural coagulant, like the synthetic coagulant, has great capacity to coagulate the raw water of the Magdalena River. However, its simultaneous use with chlorine is not recommended, because it gives rise to the production of chloroform, which is the main source of Trihalomethanes.
\end{abstract}

Keywords: drinking water, Moringa oleifera, aluminum sulfate, Trihalomethanes 


\section{Introducción}

Durante el proceso de potabilización del agua, es posible que se den una serie de reacciones químicas entre el cloro utilizado en la desinfección y la materia orgánica presente en el agua tratada. Estas reacciones dan origen a una serie de subproductos de diferente toxicidad y cancerogenecidad (Meléndez et al., 2015), conocidos como SPD (productos secundarios de cloración), que incluyen los Trihalometanos (THM), Cloroformo, entre otros. Al igual que los coagulantes sintéticos, las semillas de Moringa oleífera muestran excelentes propiedades coagulantes, constituyendo una alternativa para la potabilización de las aguas en países en vías de desarrollo, incluyendo a Colombia.

El propósito de este trabajo es Cuantificar la producción de sustancias químicas nocivas (Trihalometanos) en el agua potable tratada con coagulantes natural y sintético.

\section{Metodología}

\subsection{Muestras de agua cruda.}

Las muestras de agua cruda se tomaron sobre la margen Izquierda del río Magdalena, cerca de la captación de la planta potabilización del Municipio de Magangué, Colombia. Se realizaron dos muestreos simples, que cubrieron la época seca y lluviosa de la región, durante los meses de Agosto y Octubre de 2017, respectivamente. Se transportaron hasta el laboratorio de Suelos y Agua de la Universidad de Sucre en la ciudad de Sincelejo.

\subsection{Preparación de coagulantes.}

\subsubsection{Semilla de Moringa oleífera.}

Se obtuvieron las semillas de Moringa Oleífera en cosecha del departamento de Córdoba. Se descascararon manualmente y luego se secaron 38 poniéndolas al sol durante ocho días a temperatura ambiente. Las semillas secas se pasaron por un molino manual y se tamizó en una malla $\mathrm{N}^{\circ} 100$ en repetidas ocasiones hasta obtener un polvo muy fino. Luego $10.0 \mathrm{~g}$ del polvo obtenido se disolvió en un matraz aforados, hasta 1.0 L con solución salina de cloruro de sodio $1.0 \%$ (w / v). La solución se mezcló con agitación magnética durante 1 hora y se filtró al vacío con papel filtro de celulosa. El filtrado se rotuló como extracto coagulante salino con concentración de $10,000 \mathrm{mg} \mathrm{L}^{-1}$ y se conservó refrigerado a $4{ }^{\circ} \mathrm{C}$ hasta su aplicación (Feria et al., 2016 ).

\subsubsection{Sulfato de Aluminio tipo B.}

El Sulfato de Aluminio tipo B- $\mathrm{Al}_{2}\left(\mathrm{SO}_{4}\right)_{3} \cdot 14 \mathrm{H}_{2} \mathrm{O}$, se obtuvo comercialmente donado por la empresa de acueducto del Municipio de Magangué, Bolívar, el cual fue preparado a las mismas concentraciones que las soluciones de Moringa oleifera.

\subsection{Test de jarras}

A cada muestra de agua cruda se aplicó extracto de semillas de Moringa oleífera y Sulfato de aluminio como coagulantes, en dosis de $15,30 \mathrm{y}$ $60 \mathrm{mg} \mathrm{L}^{-1}$. Se utilizó un equipo de test de jarras marca EyQ F6-300-T equipado con seis paletas giratorias e igual número de vasos de precipitados de $1000 \mathrm{~mL}$. La mezcla rápida fue de $200 \mathrm{rpm}$ durante 1 minuto, seguido por la mezcla lenta de $40 \mathrm{rpm}$ durante 20 minutos y con un tiempo de sedimentación de 30 minutos (Feria et al., 2016) .

Se utilizó un blanco para verificar la actividad de los coagulantes naturales (AC) y la remoción de la turbidez (RT) en todos los ensayos de Jarras. Esto fue calculado usando la siguientes ecuaciones (Feria et al., 2018; Rodiño et al., 2015 ; Feria et al., 2016 ; Antov et al., 2012): 


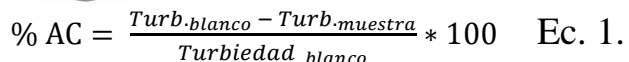

$\% \mathrm{RT}=\frac{\text { Turbidez final }- \text { Turbidez } \text { inicial }}{\text { Turbidez Inicial }} * 100 \quad$ Ec. 2.

\subsection{Equipo y parámetros fisicoquímicos}

Se midieron por duplicado turbidez y $\mathrm{pH}$ para todas las muestras de agua antes y después de los test de jarras. La turbidez fue medida con un turbidímetro Thermo Orion AQ 3010 y el pH con un pH-metro SI Analytics-Lab865, siguiendo los protocolos de medición establecidos en los métodos normalizados para el análisis de agua potable y residual según la American Public Health Association .

\subsection{Cuantificación de sustancias químicas nocivas (Trihalometanos) en el agua potable tratada con coagulantes naturales $y$ sintéticos.}

A las muestras con turbidez de 184 NTU se les realizó una simulación del proceso de desinfección con hipoclorito de sodio al 5,25\%, para obtener el rango de cloro residual permisible y se cuantificó los Trihalometanos en el agua tratada con cromatografía de gases, de acuerdo con metodologías estándar y en un laboratorio validado y acreditado por el IDEAM.

\subsection{Análisis estadístico}

En el análisis estadístico se aplicó análisis de varianza (ANOVA) con un nivel de significancia del 95\%, usando el software R versión Trial.

\section{Resultados}

La turbidez del agua en el río Magdalena puede variar dependiendo de la época y de la intensidad de la lluvia, como se muestra en la Tabla 1.
Tabla 1. Turbiedad y $\mathrm{pH}$ de las muestras de agua cruda del río Magdalena

\begin{tabular}{|l|l|l|}
\hline Parámetros & Epoca seca & Epoca lluviosa \\
\hline Turbidez (UNT) & 184 & 513 \\
\hline pH & 6.78 & 6.47 \\
\hline
\end{tabular}

Para ambas turbidez iniciales del agua cruda (época seca y lluviosa), el rango de aplicación de dosis óptima para lograr las mayores eficiencias de actividad coagulante y remoción, siendo la dosis de $60 \mathrm{mg} \mathrm{L}^{-1}$ más efectiva, como se muestra en la Tabla 2.

Se observa que la actividad coagulante dependió de la dosis aplicada y aumentó proporcionalmente conforme con el aumento de la turbidez del agua cruda. Este comportamiento es típico de la coagulación mediante adsorción y neutralización de Cargas (Rodiño., et al 2015; Ndabigengesere., et al 1995).

Tabla 2. Condiciones optima del agua del río tratada con Moringa oleífera (MO) y Sulfato de aluminio.

\begin{tabular}{|l|c|c|c|c|}
\hline \multirow{2}{*}{ Parámetros } & \multicolumn{2}{|c|}{ Época Seca } & \multicolumn{2}{c|}{ Época Lluviosa } \\
\cline { 2 - 5 } & $M O$ & Sulfato & MO & Sulfato \\
\hline Dosis $(\mathrm{mg} / \mathrm{L})$ & 60 & 60 & 60 & 60 \\
\hline Turbidez $(U N T)$ & 4.48 & 0.71 & 3.35 & 4,67 \\
\hline$p H$ & 6.9 & 5.8 & 6.82 & 5.46 \\
\hline $\begin{array}{l}\text { Actividad } \\
\text { coagulante (\%) }\end{array}$ & 95.93 & 99.3 & 98.91 & 98,7 \\
\hline Remoción (\%) & 97.56 & 99.61 & 99.35 & 99.09 \\
\hline
\end{tabular}

La Moringa oleífera, en época seca de la región, se obtuvo menor turbidez decantada de 4,48 UNT, con actividad coagulante de $95,93 \%$ y remoción de $97,56 \%$, y en época lluviosa se obtuvo menor turbidez decantada de 3,35 UNT, con actividad coagulante de $98,91 \%$ y remoción de $99,35 \%$. Datos similares se obtuvieron con el 
coagulante artificial. Sin embargo, la Moringa oleifera puede presentar ventajas sobre el sulfato de aluminio ya que estudios realizados (Jahn, 1988) han demostrado que al utilizar Moringa oleifera no quedan residuos tóxicos en el agua tratada que pudieran afectar el organismo humano. En este ensayo se logra la clarificación del agua en las tres primeras fases del proceso de potabilización (coagulación, floculación, sedimentación, filtración y desinfección). La filtración ayudará a mejorar la remoción del material suspendido en el agua y posiblemente a cumplir con el valor máximo aceptado de turbidez propuesto por la normatividad ambiental colombiana de calidad del agua.

Tabla 3. Cuantificación de Trihalometanos en el agua tratada con Moringa oleífera (MO) y Sulfato de aluminio:

\begin{tabular}{|l|l|l|l|}
\hline $\begin{array}{l}\text { Trihalometanos } \\
(\mathrm{mg} / \mathrm{L})\end{array}$ & $\begin{array}{l}\text { Agua } \\
\text { cruda }\end{array}$ & MO & $\begin{array}{l}\text { Sulfato } \\
\text { de } \mathrm{Al}\end{array}$ \\
\hline Cloroformo & $<0.005$ & 1.470 & 0.14 \\
\hline Bromodiclorometano & $<0.005$ & 0.08 & 0.08 \\
\hline Bromoformo & $<0.005$ & 0.04 & 0.02 \\
\hline ClorodiBromometano & $<0.005$ & 0.06 & 0.02 \\
\hline
\end{tabular}

La tabla 3 muestra que el agua tratada con coagulante natural superó el máximo permitido de Trihalometanos, de acuerdo con la normatividad sanitaria y ambiental vigente en Colombia (0,2 ppm para THM's), infiriendo que el uso del extracto acuoso de Moringa oleífera como coagulante presenta desventajas con respecto a la materia orgánica proveniente de la semilla que se libera en el agua durante el tratamiento, aumentando muchas veces la demanda química de oxígeno (DQO) (Eman et al.,

2014) y el carbono orgánico disuelto (DOC) en el agua tratada (Sánchez et al., 2010).

\section{Conclusiones}

Los resultados de este estudio demuestran que el coagulante natural, al igual que el sintético, tiene gran capacidad de coagulación del agua cruda del río Magdalena. Sin embargo, no es recomendable su uso simultáneo con cloro, porque da lugar a la producción de cloroformo, que es el compuesto más representativo del grupo de compuestos conocidos como Trihalometanos totales, por lo que existe un riesgo de efectos adversos a la salud.

\section{Agradecimientos}

El autor agradece a la Universidad de Sucre por su valiosa contribución en este proyecto.

\section{Referencias Bibliografícas}

Antov, M.G., Sciban, M.B. and Prodanovic, J.M. (2012) Evaluation of the Efficiency of Natural Coagulant Obtained by Ultrafiltration of Common Bean Seed Extract in Water Turbidity Removal. Ecological Engineering, 49, 48-52. http://dx.doi.org/10.1016/j.ecoleng.2012. 08.015

Eman, N; Tan,C; y Makky, E. (2014). Impact of Moringa oleifera Cake Residue Application on Waste Water Treatment : A Case Study, J. Water Resour. Prot. 6, 677-687. https://doi.org/10.4236/jwarp.2014.6706 5 .

Feria JJ; Tavera MJ, and Perna O. (2018). Extraction and efficiency of Chitosan from Shrimp Exoskeletons as coagulant for lentic water bodies. International Journal of Applied Engineering 
Research. January, 13 (2), pp. 1060-1067. https://doi.org/10.17485/ijst/2018/v11i1 $1 / 121533$

Feria, J.J., Polo, L., y Hernandez, E. (2016). Evaluación de lodos de coagulación de agua cruda tratada con Moringa oleífera para uso agrícola. Ingeniería e Investigación 36(2), 14-20, https://doi.org/10.15446/ing.investig.v36 n2.56986

Jahn S. (1988). Using Moringa seeds as coagulants in developing countries. J Am Water Works Assoc;80:43-5. https://onlinelibrary.wiley.com/doi/abs/1 0.1002/j.1551-8833.1988.tb03052.x

Meléndez Gélvez I., Pardo Perez E., Quijano Parra A. Actividad genotoxica de aguas antes y después de clorar en la planta de potabilización Empopamplona. Revista Bistua.2015.13(2):12-23

Ministerio de la Protección Social. Ministerio de Ambiente, Vivienda y Desarrollo Territorial. (2007). Resolución 2115. Bogotá: El Ministerio. Recuperado en http://www.minambiente.gov.co/images/ GestionIntegraldelRecursoHidrico/pdf/ normativa/Res_2115_de_2007.pdf

Ndabigengesere, A.; Narasiah, K. S. y Talbot B. G. (1995). Active agents and mechanism of coagulant of turbid waters using Moringa oleifera. Water Research, 29(2), 703-710. https://doi.org/10.1016/00431354(94)00161-Y

Rodiño, J.P., Feria, J.J., Paternina, R.D., y Marrugo, J.L. (2015). Sinú River raw water treatment by natural coagulants.
41

Revista Facultad de Ingeniería, Universidad de Antioquia, 76, 90-98. https://doi.org/10.17533/udea.redin.n76a 11.

Sánchez-Martín, J; Ghebremichael, K; y Beltrán-heredia, J. (2010). Bioresource Technology Comparison of single-step and two-step purified coagulants from Moringa oleifera seed for turbidity and DOC removal, Bioresour. Technol. 101 (2010) 6259-6261. https://doi.org/10.1016/j.biortech.2010.0 2.072

*Para citar este artículo:

Sampayo Benavides CH., Feria Diaz JJ

Preliminary determination of by-products of chlorination (SPD), in raw waters of the river Magdalena treated with coagulant natural and synthetic. Revista Bistua.2017.15(2):37-42

+ Autor para el envió de correspondencia y la solicitud de las separatas: Sampayo Benavides $\mathrm{CH}$. Universidad de Sucre-SUE CARIBE. csampayob@gmail.com

Recibido: Octubre 20 de 2016

Aceptado: Enero 29 de 2017 


\section{Curriculos}

C.H. Sampayo Benavides, Quimico Farmaceutico (2003) de la Universidad de Cartagena; Especialista en Ingenieria Sanitaria y Ambiental (2007) de la Universidad de Cartagena; Candidata a Magister en Ciencias Ambientales, SUE CARIBE-Unisucre. Magangué; Integrante activo del Grupo de Investigación en Medio Ambiente y Aguas (GIMAGUAS) de la Universidad de Sucre, Colombia. https://orcid.org/0000-0001-5883-513X

J.J. Feria Díaz, Ingeniero Sanitario (1999) de la Universidad de Antioquia, Magister en Ciencias Ambientales (2009) de la Universidad de Córdoba, Montería. Profesor Asociado de la Universidad de Sucre, Facultad de Ingeniería; Director del Grupo de Investigación en Medio Ambiente y Aguas (GIMAGUAS) de la Universidad de Sucre, Colombia. https://orcid.org/0000-0003-1397$\underline{1546}$ 